\title{
Plant Proteins for Medical Applications
}

\section{Narendra Reddy ${ }^{1}$ and Yiqi Yang ${ }^{2 *}$}

${ }^{1}$ Department of Biological Systems Engineering

${ }^{2}$ Department of Textiles, Clothing \& Design

Plant proteins show good potential for medical applications but offer considerable challenges in fabricating biomaterials. Despite substantial efforts, especially in the last decade, to develop polymeric biomaterials, there are no polymers that are ideally suited for tissue engineering, drug delivery and other medical applications. Therefore, the quest to find new sources for biomaterials continues. Natural proteins such as collagen and silk, carbohydrates such as chitosan and cellulose and synthetic biopolymers such as poly (lactic acid) have been extensively studied for potential medical applications. Biotechnology and nanotechnology have also been widely adopted to develop regenerated and recombinant polymers for medical applications. The advent of nanotechnology and its many advantages for medical applications have led to the development of nanofibers and nanoparticles from both the natural and synthetic polymers for tissue engineering, controlled release and other medical applications. However, both natural and synthetic polymers currently available have many limitations that restrict their use for medical applications. Biomaterials developed from natural polymers do not have the desired mechanical properties for medical applications. For instance, scaffolds developed from collagen have poor hydrolytic stability and efforts to crosslink and improve the properties have not been successful to provide cytocompatible biomaterials. Although silk has excellent mechanical properties and biocompatibility, silk has slow degradation rates and it is difficult to dissolve and process silk into various types of biomaterials. Biomaterials developed from most synthetic polymers have the biocompatibility and mechanical properties but their degradation into toxic substances in the body is a cause for concern. Similarly, metal and ceramic based biomaterials do not have the desired degradability and are difficult to process into different forms of biomaterials.

Proteins offer several advantages over carbohydrates and synthetic polymers for medical applications. Proteins are hydrophilic and as a major part of the human bodyare more cytocompatible and biodegradable. Proteins contain a number of functional groups that makes proteins carry different charges under various physiological conditions and are therefore more amenable to chemical modifications and suitable to deliver different types of drugs. Currently, collagen and silk are the two main proteins used for medical applications. However, both collagen and silk have limitations that restrict their use. Plant or cereal proteins such as zein from corn, wheat and soyproteins are more readily available than collagen and silk but are relatively unexplored for medical applications. Nevertheless, studies have been done to develop biomaterials including micro and nanofibers, films, hydrogels and micro and nanoparticles from the plant proteins for tissue engineering and medical applications. Proteins such as zein dissolve in ethanol solutions and are easily made into various types of biomaterials. Both in vitro and in vivo studies have demonstrated that zein based biomaterials are biocompatible and suitable for tissue engineering and controlled delivery. Among wheat proteins, wheat gliadin is reported to be cytotoxic whereas wheat glutenin has shown promise for tissue engineering and controlled release applications. Studies have shown that hydrogels, films and fibers made from soyproteins have good potential to be used as biomaterials. The $\mathrm{pH}$ responsiveness of proteins has been used to develop smart hydrogels from soyproteins.

Plant proteins show potential to overcome the limitations of polymers currently in use for medical applications and provide biomaterials suitable for tissue engineering, drug delivery and other medical applications. However, unlike zein, wheat gluten and soyproteins do not dissolve in common solvents and it is difficult to fabricate nanofibers and micro and nanoparticles from these proteins. Biomaterials developed from plant proteins may not have the mechanical properties required for medical applications. Approaches such as crosslinking with cytocompatible crosslinkers are necessary to make plant protein based biomaterials suitable for medical applications. Further research, especially in vivo studies using soy and wheat protein based biomaterials are necessary before plant proteins can be considered as candidates to replace the polymers in current use for medical applications.
*Corresponding author: Yiqi Yang, University of Nebraska-Lincoln, 226 HECO Bldg, Lincoln, NE 68583-0802, Tel: 402-472-5197, Fax: 402-472-0640, E-mail: yyang2@unl.edu

Received February 01, 2011; Accepted March 29, 2011; Published March 31, 2011

Citation: Reddy N, Yang Y (2011) Plant Proteins for Medical Applications. J Microbial Biochem Technol 3: i-i. doi:10.4172/1948-5948.100000e2

Copyright: @ 2011 Reddy N, et al. This is an open-access article distributed under the terms of the Creative Commons Attribution License, which permits unrestricted use, distribution, and reproduction in any medium, provided the original author and source are credited. 